\title{
A MEMS-Based, Ka-Band, 16-Element Sub-Array
}

Janice C. Rock, Tracy Hudson - U.S. Army Aviation and Missile Research, Development, \& Engineering Center; AMSRD-AMR-WD-UR; Redstone Arsenal, AL. 35898-5000; ph: 256-876-1426

Brandon Wolfson, Daniel Lawrence - Technology Service Corporation, Phase IV Systems Operation; 3405 Triana Blvd., Huntsville, AL. 35805; ph: 256-535-2142

Brandon Pillans - Raytheon Space and Airborne Systems, Advanced Products Center, Dallas, TX 75243; ph: $972-$ 344-3492

Andrew R. Brown - A. Brown Design, 46055 Bloomcrest Drive, Northville, MI 48167; 734-674-7869

Louis Coryell - U.S. Army Communications-Electronics Research, Development and Engineering Center, AMSRD-CER-ST-SS-TS, Fort Monmouth, NJ 07703-5203; 732-532-9210, x5632

janice.rock@us.army.mil; tracy.hudson@us.army.mil; brandon.wolfson@phaseiv.com; dan.lawrence@phaseiv.com; b-pillans@raytheon.com; Andrew.r.brown@sbcglobal.net; Louis.coryell@us.army.mil

Abstract-The Aviation and Missile Research, Development and Engineering Center (AMRDEC) has been involved in an Army Technology Objective (ATO) aimed at furthering phased arrays for both tactical seekers and communication links. The ATO has been pursuing both MicroElectroMechanical Systems (MEMS) and MMICbased phase shifters with an overall goal of $\$ 50 \mathrm{~K}$ for a tactical seeker at Ka-band and $\$ 5 \mathrm{~K}$ for a communication link at Ku-band. ${ }^{1,2}$

In a collaborative effort with the CommunicationsElectronics Research, Development and Engineering Center (CERDEC), the AMRDEC has worked to improve the maturity of Radio Frequency (RF) MEMS devices for use in phase shifters for phased arrays.

This paper shows that RF MEMS have vastly improved in reliability over the past few years. This paper presents the background and most current results of a task to implement a 16-element phased sub-array with RF MEMS-based phase shifters. The slat is centered at $33.4 \mathrm{GHz}$ and utilizes $1 / 2-$ wavelength spacing between elements. The individual elements consist of Vivaldi antennas. Taylor Weighting is applied to lower the overall sidelobes.

This paper discusses the MEMS design being produced and the current maturity of the design. It also discusses the insertion loss, power consumption, linearity and reliability of the phasing network.

\section{TABLE OF CONTENTS}

1. INTRODUCTION

2. SWITCH DESIGN

3. Phase Shifter Design ................................................ 2

4. PHASEd ARRAY T/R ASSEMbly ................................ 4

5 LABORATORY EVALUATION ....................................... 9

6 Discussion ANd ConClusion ...................................9

REFERENCES ..............................................................9

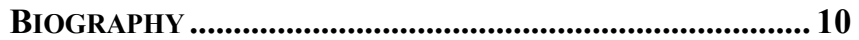

\footnotetext{
${ }^{1}$ U.S. Government work not protected by U.S. copyright

${ }^{2}$ IEEEAC paper \#1042, Version 5, Updated 2008:12:02
}

\section{INTRODUCTION}

In 2006, CERDEC issued a Broad Agency Announcement requesting proposals for Affordable RF Micro ElectroMechanical Switch (RF MEMS) Phase Shifters for Phased Arrays Manufacturing Technology (ManTech) Objective (APSPA MEMS) Program. The objective is to develop a volume production facility for reliable, low cost MEMS phased shifters for use in phased arrays for both active and passive missile seekers and for on-the-move SATCOM communications systems for the Warfighter. AMRDEC has been involved in this program since its inception and early Calender Year 2008 (CY2008) initiated an effort to demonstrate the results of the CERDEC MTO program in a 16-element, Ka-band, MEMS-based electronically-steerable slat.

A major contributor to both the cost and loss of these system types, particularly in the passive systems, is the packaged Monolithic Microwave Integrated Circuit (MMIC) phase shifter. MEMS phase shifters offer a low cost $(<\$ 10)$, low loss alternative to these costly components. The successful completion of this ManTech program should result in an overall cost reduction of up to $25 \%$ for phased array antennas operating in the $\mathrm{Ku}, \mathrm{K}$, and $\mathrm{Ka}$ frequency bands. In addition, MEMS-based phase shifting promises to reduce the loss by $50-66 \%$ compared to MMICbased phase shifting.

The prime contractor, Raytheon Space and Airborne Systems, has produced a 4-bit packaged phase shifter demonstrating approximately 2.5 to $3.0 \mathrm{~dB}$ of loss. The switch and phase shifter design are discussed in this paper.

To demonstrate the promising results of the ManTech program, AMRDEC has implemented a task to demonstrate electronic beam steering at Ka-band, using a 16 element Vivaldi antenna slat array. This paper presents the results of that task to date. The remaining laboratory evaluation of the slat will be included in the final paper if completed in time, and/or presented at the conference. 


\section{SWITCH DESIGN}

In 1995, Raytheon pioneered the development of RF MEMS technology for microwave and millimeter-wave applications with the development of the first capacitive RF switch [1]. Since then, Raytheon has been involved in the design and development of high-performance RF MEMS for advanced phased-array applications. Raytheon has developed an RF MEMS switch that is optimized for low RF insertion loss, high switching speed, high-power handling, excellent temperature stability, and long cycle lifetime (Table 1). With support from the Army, DARPA, ONR, and AFRL, Raytheon has demonstrated low-loss, multi-bit phase shifters, routers, and digitally tunable filters across the entire 0.1 to $50 \mathrm{GHz}$ frequency range. A picture of the basic switch is shown in Figure 1.

Table 1. RF MEMS switch performance summary.

\begin{tabular}{|l|l|}
\hline \multicolumn{2}{|l|}{$\boldsymbol{R} \boldsymbol{F}$ MEMS Switch Performance } \\
\hline Insertion loss at $40 \mathrm{GHz}$ & $<0.07 \mathrm{~dB}$ \\
\hline Isolation at $40 \mathrm{GHz}$ & $>25 \mathrm{~dB}$ \\
\hline Temp Stability & $-55^{\circ} \mathrm{C}$ to $+85^{\circ} \mathrm{C}$ \\
\hline Switching speed & $<5 \mu \mathrm{s}$ \\
\hline Intercept point & $>+87 \mathrm{dBm}$ \\
\hline Switching voltage & 30 volts \\
\hline Reliability & $>10^{11}$ cycles \\
\hline Size & $280 \times 120 \mu \mathrm{m}$ \\
\hline
\end{tabular}

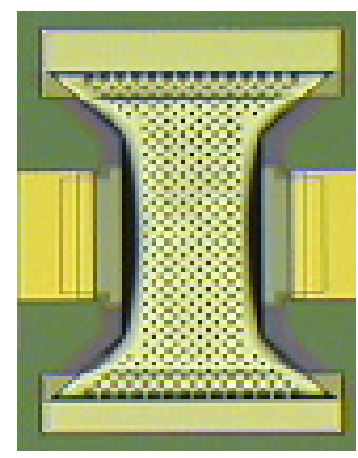

Figure 1. Top-view of the individual RF MEMS switch.

Raytheon's latest switch design achieves over 200 billion operating cycles without failure with a switching speed under 5 microseconds and power consumption under 1 microwatt. Innovations in switch membrane materials and device structure now provide much greater thermal stability, and prototype switches operate from $-55^{\circ} \mathrm{C}$ through $+85^{\circ} \mathrm{C}$ with actuation voltage change of only a few volts.

Typical switch insertion loss is less than $0.1 \mathrm{~dB}$ at frequencies through $40 \mathrm{GHz}$. These switches are constructed using only metals and dielectrics, and detailed measurements of Raytheon's RF MEMS switches show no observable RF nonlinearity to intercept points (TOI) as high as $+87 \mathrm{dBm}(5,000$ watts $)$ [2].

\section{Packaging}

Like all micromechanical systems, RF MEMS switches are subject to damage from corrosion and contamination from exposure to dust, air, and moisture. Encapsulating the switches in a particle-free, hermetic package eliminates failures due to these environmental effects. To meet operational requirements, the package must be compact and not degrade RF circuit performance. The challenge is to provide this protection at very low cost in a high volume production environment. Over the past three years, Raytheon has developed a highly innovative wafer-level hermetic packaging approach [3] that meets all requirements for reliability, operating temperature range, yield, and cost. This near-hermetic package (Figure 2) adds very little to the total circuit price.

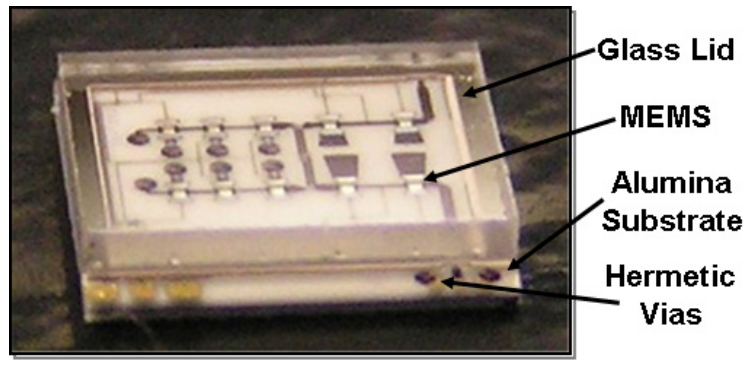

\section{Figure 2. Photograph of near-hermetic wafer-scale packaged RF MEMS.}

Maturity

Under the Army's Affordable RF Micro-ElectroMechanical Switch (RF MEMS) Phase Shifters for Phased Arrays Manufacturing Technology Objective (APSPA MEMS) Program, Raytheon is currently fabricating RF MEMS phase shifters [4] in its Advanced Multilayer Interconnect (AMI) facility, an ISO 9001-qualified manufacturing environment that has fabricated more than 500,000 comparable circuits. The APSPA Mantech program is a three-year, $\$ 5 \mathrm{M}$ effort aimed at raising the TRL and MRL levels of Raytheon's RF MEMS technology to 8 for system insertion. The program is currently in its third year with much success. Included in this effort are improvements in reliability $\left(10^{12}\right)$, RF power handling ( $2 \mathrm{~W}$ hot), operating temperature range $\left(-55\right.$ to $\left.+85^{\circ} \mathrm{C}\right)$, switching speed $(<5 \mu \mathrm{s})$ and cost. By leveraging the Army's RF MEMS Mantech program investment, Raytheon is able to provide low-loss, highly reliable switches for many applications including low-cost phased arrays.

\section{Phase Shifter Design}

The phase shifter design is based on a class II loaded-line 
topology [5, 6]. This topology was a compromise of simplicity, low-loss, compact size, and fit well with the equivalent model of the MEMS switch. The class II loaded line consists of a pi-network of a switched shunt reactance, a series transmission line of length L1_eff, and impedance Z1_eff as shown in Figure 3. Full theory and design examples of this concept is well documented in [5] and not detailed here. For the implementation used in this system, the switched reactance consists of the MEMS switch followed by a shunt radial stub [7]. The zero state consists of reactive loading of the switch up-state followed by the radial stub. The phase shifted state uses the down state switch capacitance and the radial stub.

One of the major difficulties with a loaded line topology is obtaining large phase shifts while maintaining bandwidth. Typically, $45^{\circ}$ is the largest state implemented with a loaded-line topology [6]. Higher phase shifts are typically done by cascading $45^{\circ}$ sections. However, even the $45^{\circ}$ stage proved difficult to implement and meet bandwidth requirements with the required loaded line and the Con/Coff ratio at Ka-band. Therefore, even multiple stages were used for the $45^{\circ}$ bit. To further reduce the number of stages required in cascaded bits, ripple was introduced. For the $90^{\circ}$ and $180^{\circ}$ stages, the phase shift per section and the overall impedance values were adjusted to provide an equal ripple response. This has the effect of allowing one to reduce the number of cascaded sections while still maintaining bandwidth when two or more stages are used. This allowed the $90^{\circ}$ stage to be implemented with as few as two sections and the $180^{\circ}$ with only 4 stages. However, the out-of-band performance of these stages rolls off much faster than conventional cascaded sections.

The package is based on a hermetic wafer scale package with $20 \mathrm{~mm}^{2}$ exterior dimensions. In addition to having to meander the phase shifter through the package interior, the transitions in and out of the package had to be optimized for this operating band. Due to the high capacitance of the feedthrough line to the grounded seal ring, the feeds were made very inductive to attempt to tune out some of the shunt capacitance. An optimization was performed to minimize in-band insertion loss from the package transition. Figure 4 shows the resulting, packaged phase shifter. Measurements of isolated package transitions show $<0.2$ $\mathrm{dB}$ insertion loss.

The fabricated and packaged phase shifter were measured. All data shown is from an average, fully packaged phase shifter with no de-embedding performed. There was a slight shift in performance from the designed values, primarily shown in the $22.5^{\circ}$ and $45^{\circ}$ bits. Both of these bits show excessive phase shift compared to the designed values. This can be fixed in future designs and is not a limitation of the technology. Even with the long $22.5^{\circ}$ and $45^{\circ}$ states, the RMS phase error at $33 \mathrm{GHz}$ is only $8.8^{\circ}$. The total insertion loss at $33 \mathrm{GHz}$ is $2.5 \mathrm{~dB}$ average with an RMS amplitude error $0.3 \mathrm{~dB}$.
Even though the RMS phase error is high, primarily due to the $22.5^{\circ}$ and $45^{\circ}$, the presented phase shifter shows vastly reduced insertion loss compared with other MMIC technologies such as the Triquint TGP2102 5-bit phase shifter ( $7 \mathrm{~dB}$ nominal insertion loss). The results of the laboratory evaluation of the packaged MEMS phase shifters are presented in Figures 5, 6 and 7.
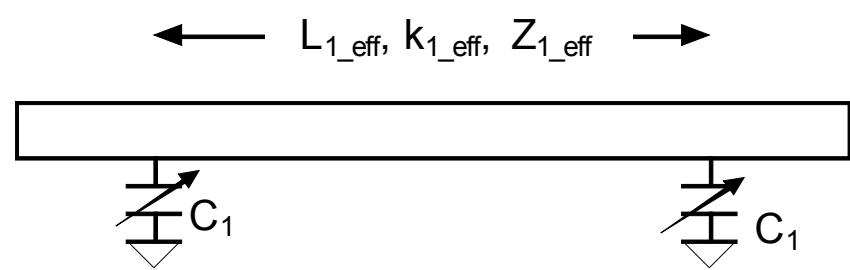

Figure 3. Topology of a capacitively loaded Class II loaded-line phase shifter.

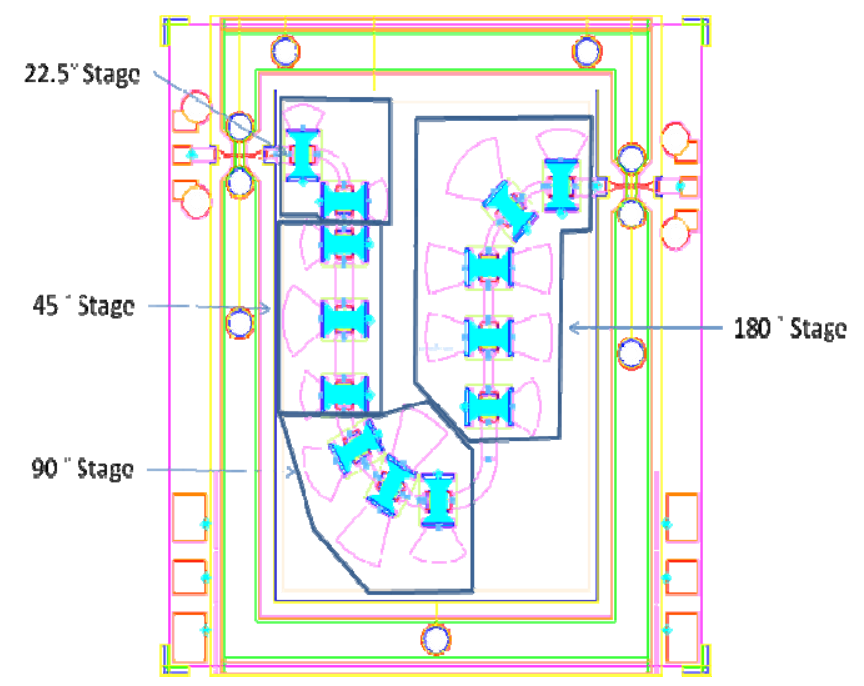

Figure 4 - Layout of the Ka-Band phase shifter.

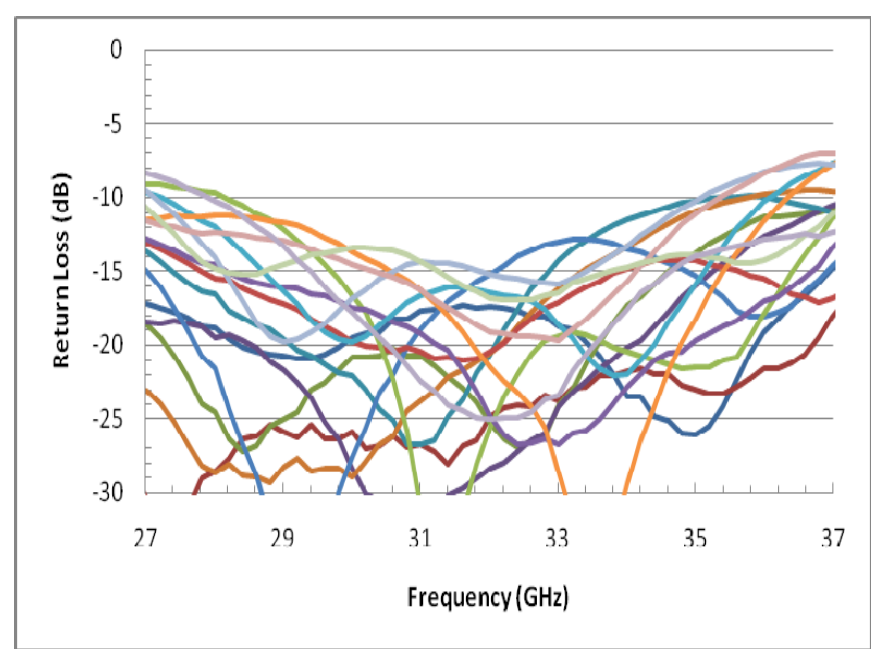

Figure 5. Measured Return Loss. 


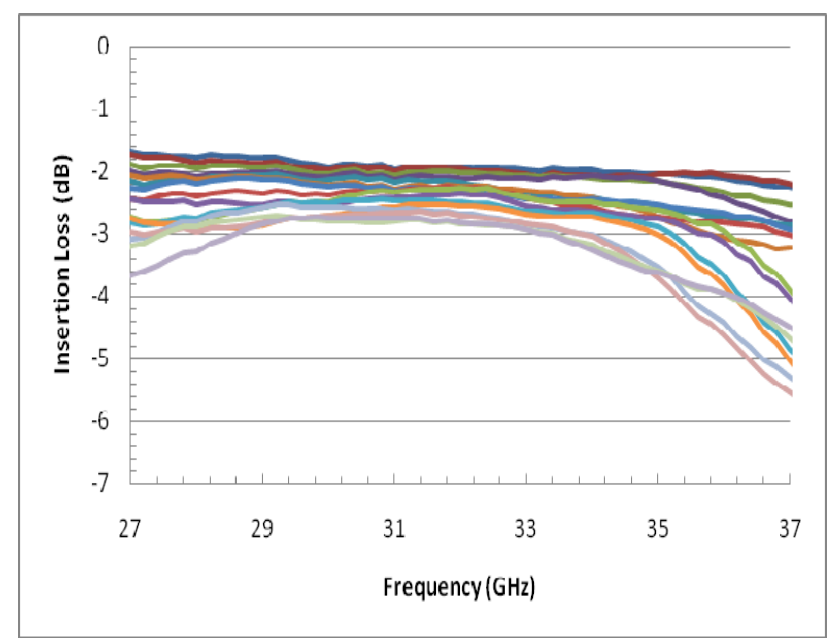

Figure 6. Measured Insertion loss of all 16 states.

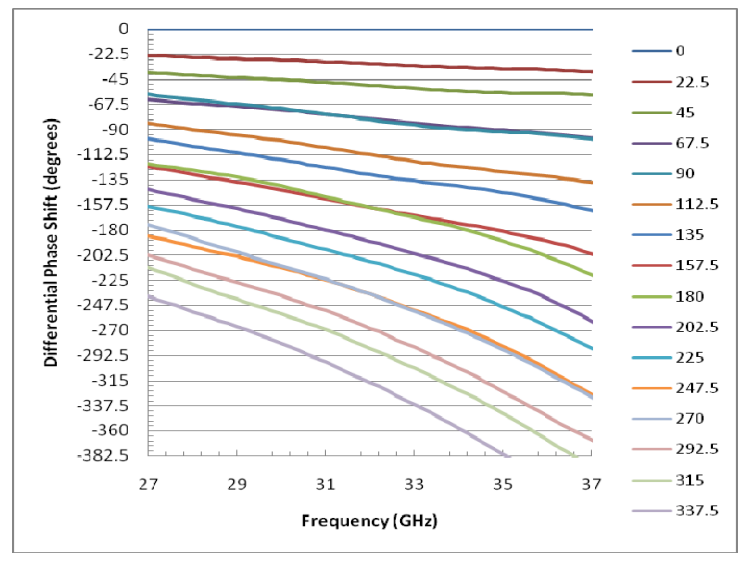

Figure 7. Measured Phase Shift.

\section{Phased Array T/R Assembly}

The slat assembly is composed of the microwave antenna slat module mated to a control and interface module. A block diagram of the slat assembly is shown in Figure 8. The microwave antenna slat module contains all of the assembly's microwave circuitry as well as the circuitry necessary to support the operation of the microwave devices, such as DC regulators, power sequencing circuits, switch drivers and high voltage multiplexers. The high voltage multiplexers are used to deliver the high voltage control signals, necessary to actuate the MEMS switches in the phase shifters, to the appropriate inputs of the MEMS phase shifters. The control and interface module provides the user with a simple method of controlling and interfacing with the microwave antenna slat module

\section{Microwave Antenna Slat Module}

The microwave antenna slat module implements 16 Vivaldi antennas and a 16-way 20dB Taylor weighted power divider to drive the MEMS phase shifters at the inputs to the antennas. A transmit/receive circuit made up of Ka-band MMIC amplifiers and switches is utilized at the input of the power divider to boost the transmitted/received signals. The Vivaldi antenna was chosen for its broadband performance and ease of implementation in a microstrip printed circuit design. The antenna design was finalized and its performance simulated using HFSS 3D electromagnetic modeling software. The 16-way power divider implements the $20 \mathrm{~dB}$ Taylor weights by cascading unequal power dividers. The design of the power divider was completed and modeled using Microwave Office simulation and design software. The circuits used to control the operational states of the Tx/Rx circuitry as well as the phase states of the 16 MEMS phase shifters are located on the backside of the multilayer board used to implement the microwave circuitry. These circuits on the backside of the microwave antenna module are controlled by signals output from the control and interface module.

\section{Antenna Design}

Vivaldi antennas were first introduced as a new class of frequency independent antennas that produce a travellingwave through an exponentially tapered slot [8]. These types of antennas are commonly referred to as tapered slot antennas (TSA) with many possible variations on the length and profile of the taper. A modified TSA, known as the antipodal Vivaldi antenna, was introduced later with the benefit of direct feeding by a microstrip line [9]. The antipodal Vivaldi antenna element design provides a wideband, broad radiation pattern that is well-suited for use in a phased array antenna. Another attractive feature of the Vivaldi antenna is the ease with which it can be driven with a microstrip feed network, and thus, integrated with surfacemount phase shifter components [10]. The basic radiating element is shown in Figure 9. The element is fabricated from a single substrate layer with metallization on both sides. A profile of the substrate ( 5 mil thickness, $\left.\varepsilon_{\mathrm{r}}=3.0\right)$ is shown in Figure 10. The element begins by exponentially tapering the ground plane of the microstrip feed line until the width is the same as the signal line. For a good impedance match, the taper extends for about onewavelength distance. At this point the ground plane and signal lines are flared symmetrically on opposite sides of the substrate to form a broadband dipole radiator. Tuning of the antenna is achieved by empirical adjustment of the flare geometry. 


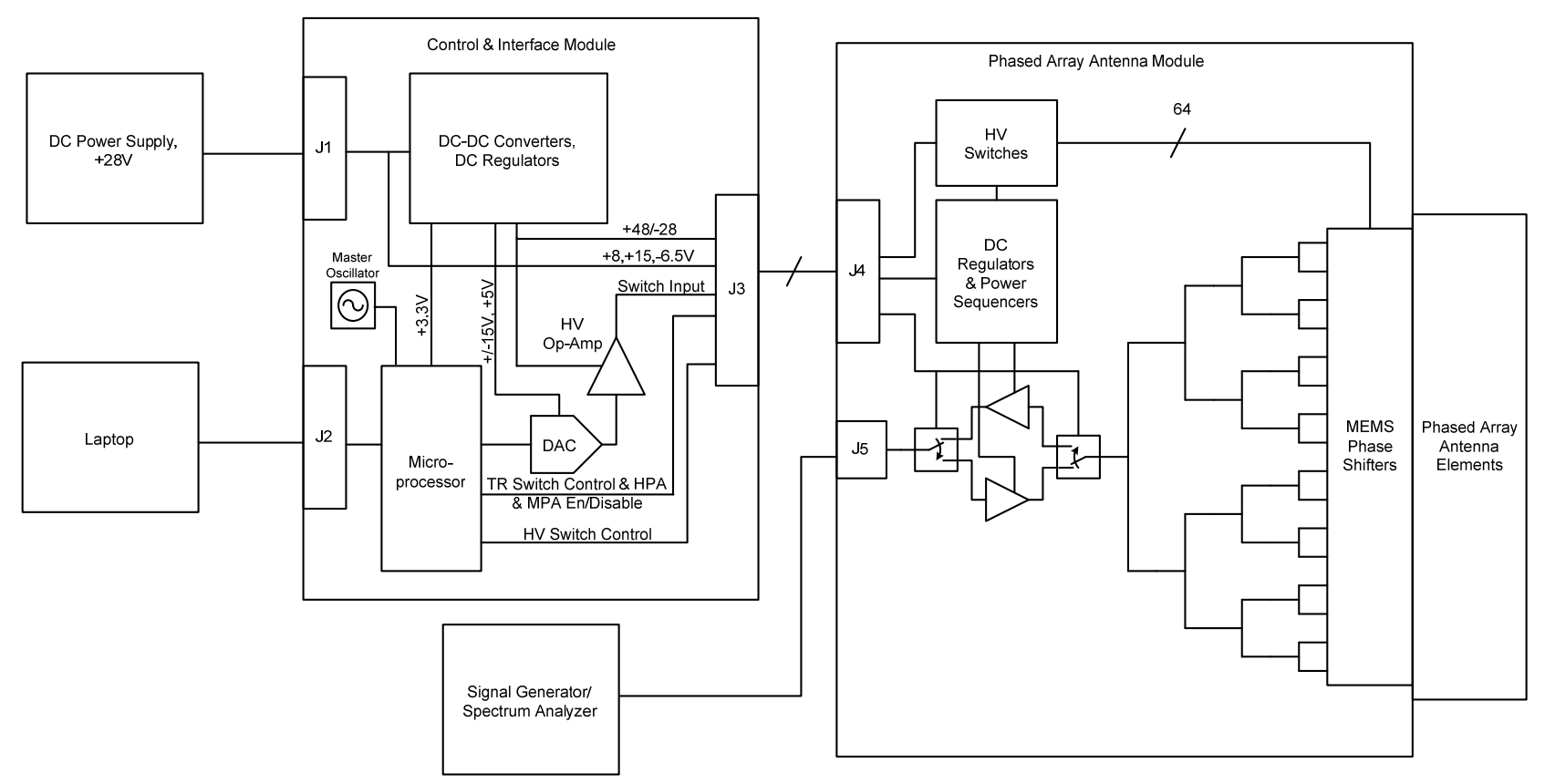

Figure 8. Block Diagram of Antenna Slat Assembly.

\section{Antenna Modeling and Simulation}

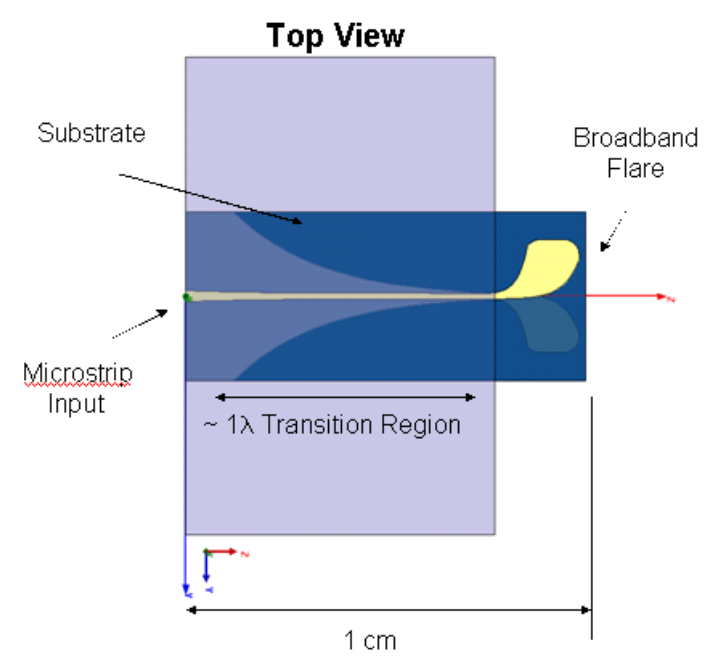

Figure 9. Antipodal Vivaldi Element Design.

Substrate Profile

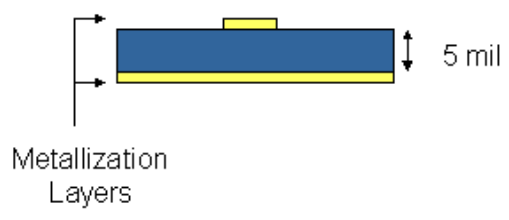

Figure 10. Substrate Profile.
A four-element subarray of Vivaldi antennas is shown in Figure 11. An HFSS simulation of the four-element subarray has been constructed in order to tune the radiating elements in the array environment. The overall size of the simulated array has been kept small to maintain reasonable run-times. Results in Figure 12 show the input match for each element in the subarray. Notice the return loss is better than $10 \mathrm{~dB}$ across the complete bandwidth, confirming the broadband nature of the Vivaldi elements. Slight variations in the resonance of each element can be attributed to the mutual coupling and parasitic loading of different elements in the array. Scanning of the subarray in the E-plane is accomplished by applying a progressive phase shift across the array. HFSS simulation results showing the array radiation pattern for several scan angles are shown in Figure 13. Uniform amplitude weighting was used for the subarray simulation. Note that scanning past $45 \mathrm{deg}$ results in increased sidelobes and a reduced mainlobe due to the rolloff of the element pattern. 


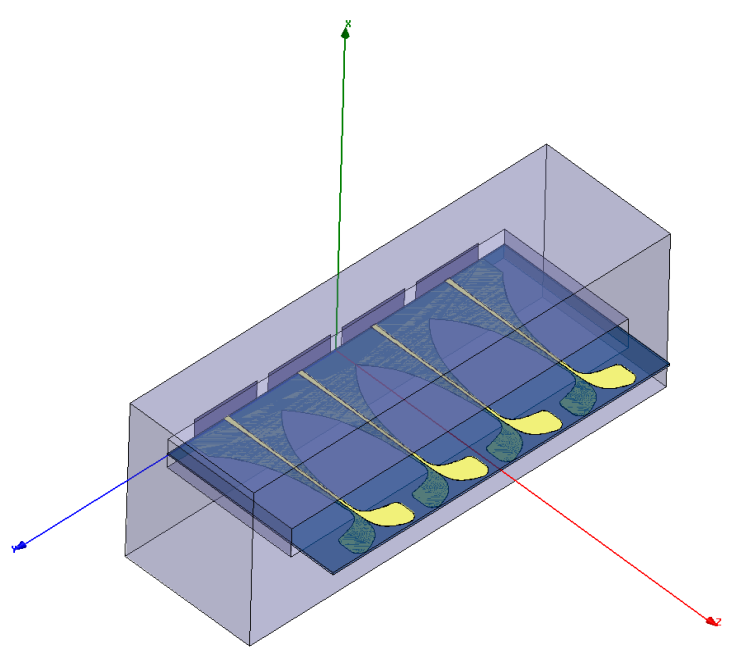

Figure 11. Four element subarray HFSS model.

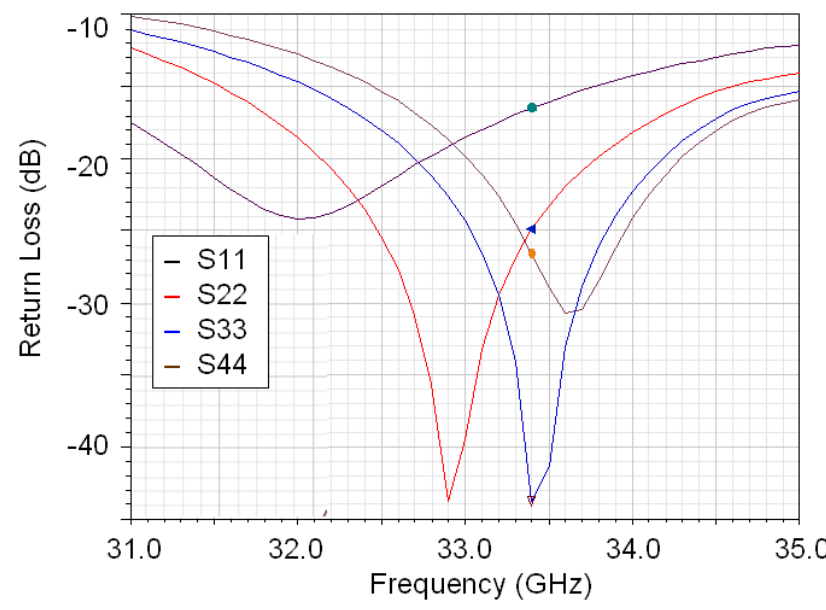

Figure 12. Input match for each element in the fourelement subarray.

$$
\beta=10^{\frac{S L L}{10}}-4.603 \frac{\sinh (\pi \beta)}{\pi \beta}
$$
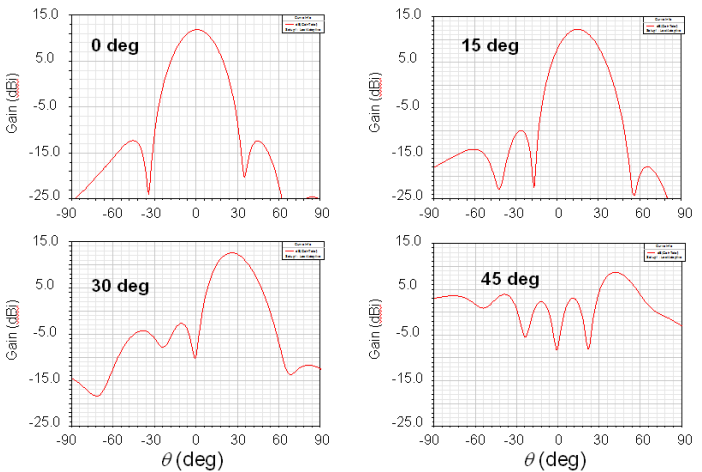

\section{Figure 13. Scanned E-plane radiation patterns for the four-element subarray.}

Power Divider

The 16-way power divider is constructed from four stages of two way power dividers. The first three stages are made by simple T-junction power dividers and the final stage of power divider utilize unequal split Wilkinson power dividers. The use of T-junction power dividers for the first three stages was done to reduce design complexity and space and to eliminate the need for isolation resistors. While the T-junction power dividers simplify the design, they do not provide isolation between the output ports. It was found that the performance of the power divider could be significantly degraded if its 16 outputs were not terminated with a load that was well matched $(\Gamma \leq-20 \mathrm{~dB})$ to the design impedance of 50 ohms. In some cases the MEMS phase shifters have been shown to have an input return loss as high as $-15 \mathrm{~dB}$ to $-10 \mathrm{~dB}$ in the band of interest which was not good enough to prevent performance degradation of the power divider. Along these same lines, the input return loss of the MEMS phase shifters can vary with changing phase shift settings causing varying performance degradation of the power divider. For these reasons, the final stage of the power divider was chosen to be Wilkinson power dividers. This provided enough port-to-port isolation to allow for the imperfect loads presented by the MEMS phase shifters.

For the design of the power divider, 20dB Taylor oneparameter weighting was used. The value of $20 \mathrm{~dB}$ was chosen to provide some reduction in sidelobe level while making the design of the power divider more feasible. The Taylor weighting coefficients can be found from (1) and (2) below.

$$
w_{n}=\frac{J_{0}\left(j \pi \beta \sqrt{1-\left(2 \frac{n-\frac{N-1}{2}}{N-1}\right)^{2}}\right)}{J_{0}(j \pi \beta)}
$$


$J_{0}$ is the modified Bessel function and $\beta$ is related to the sidelobe level and can be found by finding the root of equation (2). In (2) SLL is the desired sidelobe level in $\mathrm{dB}$.

The relative output powers at the two output ports of a twoway power divider can be defined as shown in (3). $P_{l}$ and $P_{2}$ are the relative output powers at ports 1 and 2 relative to $P_{i n}$.

$$
\begin{gathered}
P_{1}=\alpha P_{i n} \\
P_{2}=(1-\alpha) P_{i n} \quad \text { where } 0<\alpha<1
\end{gathered}
$$

Once the Taylor weighting coefficients are known, one can find the values of $\alpha$ in (3) for each of the power dividers. Since we have an even number of outputs and the Taylor weighting coefficients are symmetric the value of $\alpha$ for the first stage power divider will always be 0.5 and the values of $\alpha$ for the cascaded power dividers at each output of the first power divider will simply be mirrored copies of each other. For this design the values of $\alpha$ at each stage are shown in Table 2.

Table 2. Values of a for each power divider stage.

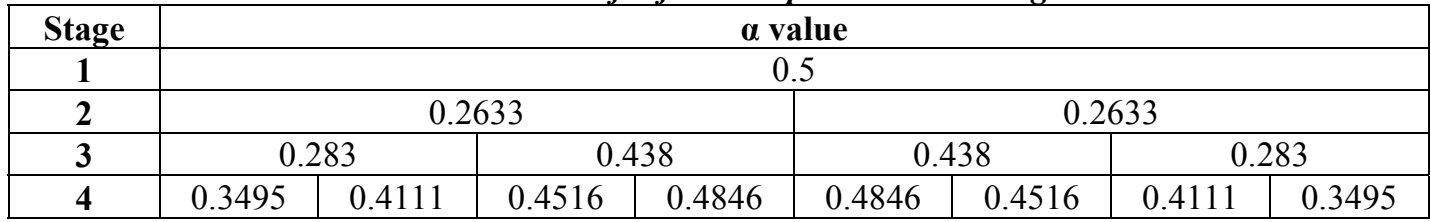

two output impedances also makes the design less trivial

For a simple T-junction power divider one can use the values of $\alpha$ above to find the necessary impedance that needs to be presented to the power divider input port by each of the two output ports to get the desired unequal power division. The relationship between the impedances at each side of the T-junction to the values of $\alpha$ is shown in (4).

$$
\begin{gathered}
Z_{1}=\frac{Z_{0}}{\alpha} \\
Z_{2}=\frac{Z_{0}}{(1-\alpha)}
\end{gathered}
$$

In (4) $Z_{1}$ and $Z_{2}$ are the impedances presented to each side of the T-junction power divider and $Z_{0}$ is the characteristic impedance of the input to the T-junction as depicted in Figure 14 where the second stage $\mathrm{T}$-junction power divider is shown. One quickly finds that the greater the sidelobe reduction the more difficult it becomes to design some of the power dividers due to the unreasonably high values of impedance needed. The high impedances can become an issue when they lead to trace widths that are too narrow for standard fabrication processes. The large ratio between the since one side of the $\mathrm{T}$-junction will require a relatively

wide trace while the other side requires relatively very narrow trace. This is the main reason that $20 \mathrm{~dB}$ Taylor weights were chosen over those with more sidelobe level reduction. One can help alleviate the need for unreasonably narrow traces by decreasing the value of $Z_{0}$ at the input to each power divider stage. This is the approach that was taken for this design as seen in Figures 14 and 15. Figure 15 shows the overall 16-way 20dB Taylor weighted power divider design. One can see that the final stage of power dividers are Wilkinson power dividers. As mentioned previously this was done to provide some channel to channel isolation to help prevent performance degradation due to the imperfect impedance match presented by the MEMS phase shifters that are fed by this power divider.

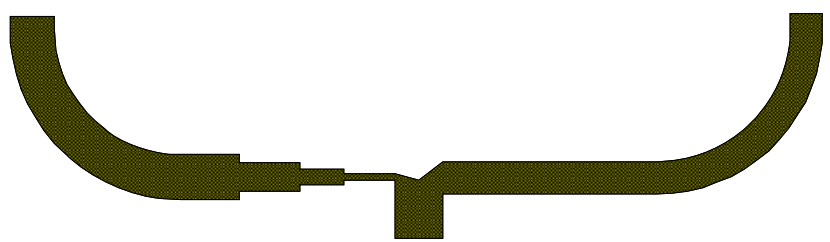

Figure 14. Second stage two-way T-junction power divider. 


\section{Modeling and Simulation}

The modeling and simulation for the power divider was completed in Microwave Office. The substrate used is the same as that used for the antenna design ( 5 mil thickness, $\varepsilon_{\mathrm{r}}$ $=3.0$ ). It can be seen from Figure 16 that the input characteristic impedances for each stage were chosen strategically to both keep the narrower line widths in the design as a reasonable width as well as remove the need for impedance transformers on one side of many of the power dividers.

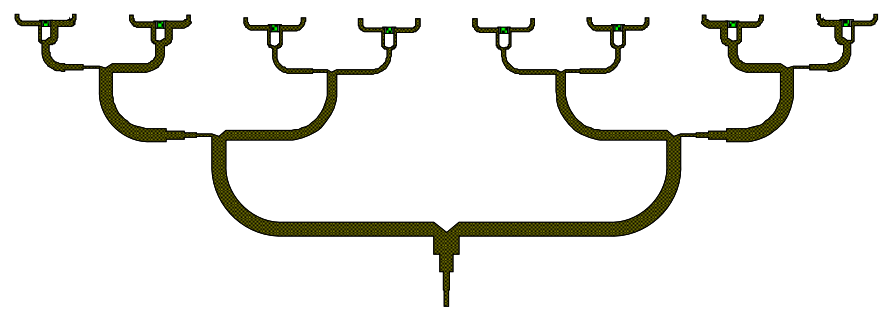

Figure 15. 16-way Taylor weighted power divider.

The simulation results for the input return loss of the 16way power divider is shown in Figure 16. Here it is seen that the power divider is well matched over the entire band of interest. Also shown in Figure 16 is the insertion loss from the input of the power divider to either of the two center most output ports. The expected insertion loss for the two center ports is $-9.72 \mathrm{~dB}$ in a loss less system.

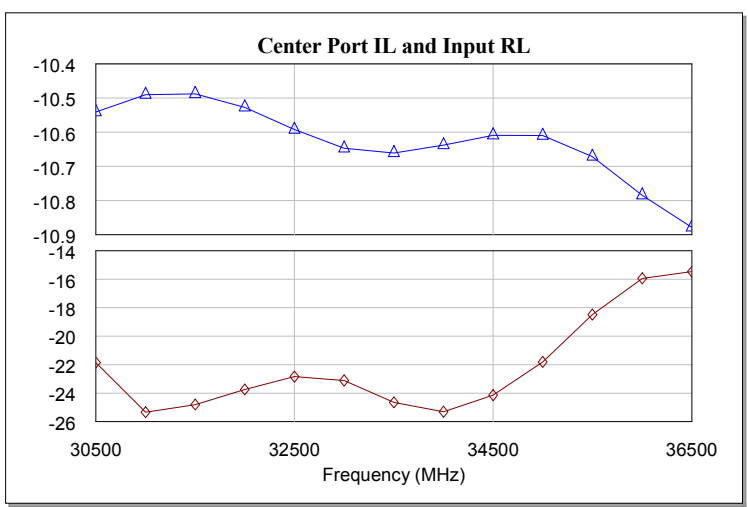

Figure 16. Simulation results for the input loss.

From the simulation it is seen that the circuit has an approximate substrate loss of $1 \mathrm{~dB}$. This is to be expected at these frequencies. For the rest of the ports it is best to look at the output power relative to the center most ports to ensure that the design effectively implements the desired Taylor weighting. Figure 17 shows this by plotting the difference between the power at the center port and the adjacent ports. In this figure the markers are placed at the center of the band of interest and the insertion loss of each port relative to the center port insertion loss of Figure 16 is shown. The values at each marker are shown in the legend. The marker $\mathrm{m} 1$ represents the port farthest from the center whereas $\mathrm{m} 7$ represents the port nearest the center port. Since the design is symmetrical we only need to look at one side of the design.

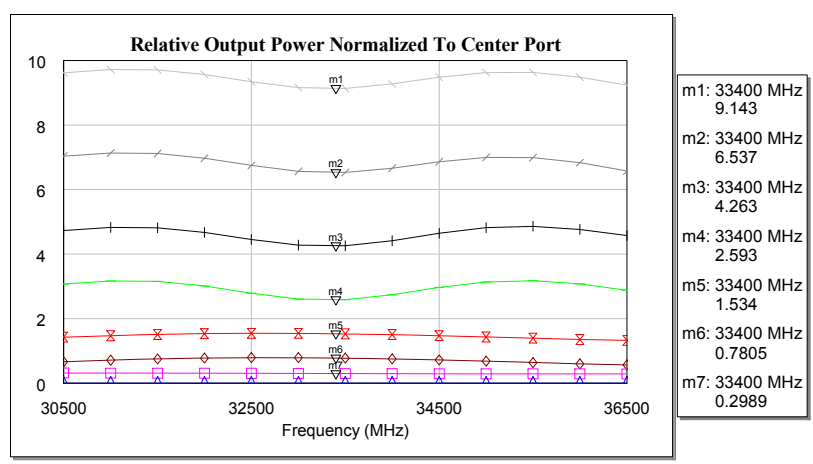

Figure 17. Plot of the difference between the power at the center and adjacent ports.

Using the Taylor weight coefficients one can find the expected relative output powers at each port. These are shown in Table 3 along side of the simulated values shown in Figure 17. In the table port numbers 1-7 are synonymous to markers m1-7 in Figure 17 and likewise represent going from the outermost port to the innermost port. In this table it is seen that the calculated and measured values are closely matched. 
Table 3. Output power at each port relative to center most port output power.

\begin{tabular}{|c|c|c|}
\hline Port \# & Calculated & Simulated \\
\hline 1 & 9.13 & 9.143 \\
\hline 2 & 6.44 & 6.537 \\
\hline 3 & 4.39 & 4.263 \\
\hline 4 & 2.83 & 2.593 \\
\hline 5 & 1.66 & 1.534 \\
\hline 6 & 0.81 & 0.7805 \\
\hline 7 & 0.27 & 0.2989 \\
\hline
\end{tabular}

\section{Control board}

The control and interface module houses the circuitry necessary to provide the control signals and various voltages to the microwave antenna subassembly. The control and interface module also provides a means for one to communicate with the phased array assembly using a standard computer interface. The circuitry mainly consists of a FPGA, a DAC, high voltage op-amps and DC-DC converters. A graphical user interface was developed to interface with the FPGA and provide the user with a method to control the various states of the phased array module. The FPGA is also used to control the DAC whose output is buffered and amplified to the high voltage levels needed to drive the MEMS phase shifters. This buffering and amplification is done by the op-amps. The outputs of the op-amps are presented to the microwave antenna subassembly which employs high voltage multiplexors on the backside of its circuit board to set the appropriate bits of the MEMS phase shifters and ultimately steer the antenna array beam pattern. The DC-DC converters allow the phased array assembly to be powered by a single 28 Volt source.

\section{LABORATORY EVALUATION}

At press time, the final design is complete and undergoing fabrication. A Ka-band compact range evaluation of the final slat will be held to collect data at multiple steering angles including a $0^{\circ}, 30^{\circ}$, and $45^{\circ}$ steer. 3 dimensional radiation plots will be generated for the steered angles of interest. Also, S-parameters of the resulting slat will be collected and reported. A frequency sweep will be conducted to generate bandwidth information.

\section{Discussion and Conclusion}

To our knowledge, this represents the first passive electronically-steerable phased array slat populated with packaged MEMS devices at Ka-band. The Raytheon MEMS-based phase shifters developed under the CERDEC MTO are significantly mature but not the only 4-bit phase shifters known to the Government at Ka-band. They were chosen for this task due to the collaborative environment with CERDEC and AMRDEC.
The final results of this effort will be reported once the task is complete. Further information on this task or on the specific elements of this task can be obtained from the authors.

\section{REFERENCES}

[1] Goldsmith, C.L.; Zhimin Yao; Eshelman, S.; Denniston, D.; Performance of low-loss RF MEMS capacitive switches Microwave and Guided Wave Letters, IEEE [see also IEEE Microwave and Wireless Components Letters] Volume 8, Issue 8, Aug. 1998 Page(s):269 271

[2] Pillans, B.; Morris, F.; Chahal, P.; Frazier, G.; JeongBong Lee; Schottky barrier contact-based RF MEMS switch, Micro Electro Mechanical Systems, 2007. MEMS. IEEE 20th International Conference on 21-25 Jan. 2007 Page(s):167 - 170IMS 2007 - Honolulu, Hawaii

[3] Abstract submitted to IMAPS 2009

[4] Pillans, B.; Coryell, L.; Malczewski, A.; Moody, C.; Morris, F.; Pillai, V.; "RF MEMS Phase Shifter Manufacturing Technology," Defense Manufacturing Conference 2008, Orlando, FL.

[5] Atwater, Harry A., "Circuit Design of the Loaded-Line Phase Shifter," IEEE Transactions on Microwave Theory and Techniques, Vol. MTT-33, No. 7, July 1985, pp. 626-634.

[6] Opp, Francis L. and Hoffman, W. F., "Design of Digital Loaded-Line Phase-Shift Networks for Microwave Thin-Film Applications," IEEE Transactions on Microwave Theory and Techniques, Vol. MTT-16, No. 7, July 1968, pp. 462-468.

[7] Rebeiz, Gabriel M., RF MEMS, Theory, Design, and Technology, John Wiley \& Sons, Inc., New Jersey, 2003, pp. 221-257.

[8] P. J. Gibson, "The Vivaldi Aerial," in Proc. 9th Eur. Microwave Conf., Brighton, U.K., Sept.. 1979, pp. 101-105. 
[9] E. Gazit, "Improved Design of the Vivaldi Antenna," IEE Proc., Part H, Vol. 135, No. 2, 1988, pp. 89-92.

[10] S. G. Kim and K. Chang, "A low cross-polarized antipodal Vivaldi antenna array for wide-band operation," in Proc. IEEE Int. AP-S Symp., Monterey, CA, Jun. 2004, pp. 2269-2272.

\section{BIOGRAPHY}

Janice $\boldsymbol{C}$. Rock is a research engineer with the RF Technology Division in the Applied Sensors, Guidance, and Electronics Directorate, Aviation and Missile, Research, Development and Engineering Center; Redstone Arsenal, AL. She has an

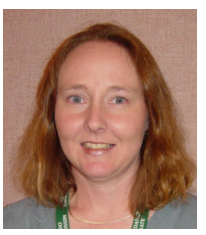
M.S.E. in Electrical Engineering from the University of Alabama in Huntsville. Her main area of research is in the field of phased array antenna systems. Other interests are in MEMS, semi-conductor antennas, and chip-level component integration issues.

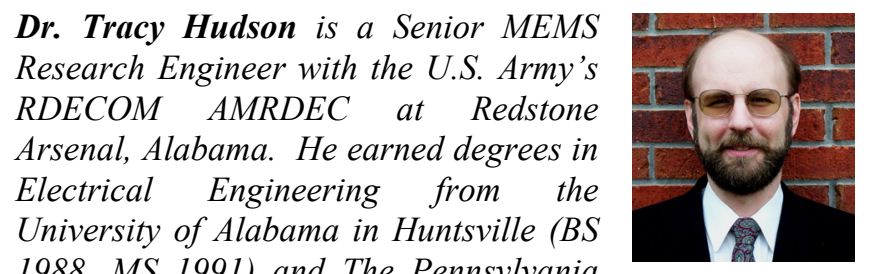
1988, MS 1991) and The Pennsylvania

State University (PhD 1997). Dr. Hudson has over 24 years experience developing component technology for Army rotorcraft and missile systems at the U. S. Army. He has been recognized for his scientific achievements on numerous occasions to include the bestowal of the U.S. Army's Research and Development Award in 1991 and 2003. Dr. Hudson has publish or presented over 80 papers within his career thus far (most being peer-reviewed journal articles). He is a member of IEEE, The Optical Society of America, and a Fellow of SPIE-The International Optical Engineering Society. His current technical interests include microfabrication process development, MEMS device prototyping (inertial, microwave, chemical sensing, acoustic sensors, piezoelectric actuators and materials, electro-optical materials, and power generation/management), nanotechnology process development, and nanotechnology applications.

Dr. Brandon Pillans graduated with a Bachelor of Science in Electrical Engineering from Texas A\&M University in 1996. He received his Master of Science in Electrical Engineering from Texas A\&M University in 1998 and his Ph.D. from the University of Texas at

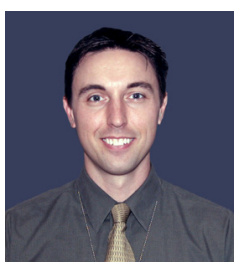
Dallas in 2006. He began working at Raytheon Systems Company in 1998 performing RF MEMS switch research and development. While in the RF MEMS group, Brandon has performed many RF MEMS MMIC designs such as a Ka-Band phase shifter that has measured world record performance in addition to tunable bandpass and bandstop filters and tunable impedance matching networks. He has also worked extensively on increasing the reliability of the capacitive RF MEMS switch, resulting in six orders of magnitude increase in switch lifetime over that period. He has written over 16 published articles on MEMS technology and holds three RF MEMS patents. Brandon is currently the technical lead of the RF MEMS group at RaytheonDallas.

\section{Brandon Wolfson is a Senior Engineer in RF and microwave design at the Phase IV Systems Operation of Technology Service Corporation (TSC), in Huntsville, Alabama. He earned his degrees in Electrical Engineering from Auburn University (BSEE 1999, MSEE 2001) where his

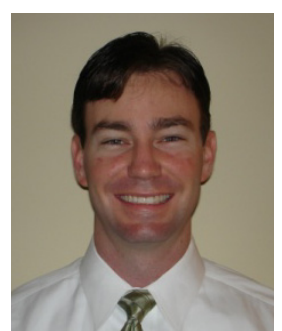
focus was in the areas of electromagnetic theory and modeling as well as microwave circuit design and simulation. Mr. Wolfson has worked as a RF and microwave design engineer for more than 7 years at TSC/Phase IV Systems and currently also serves as a program manager, system engineer, lead design engineer and employee supervisor. Mr. Wolfson has authored multiple publications as well as received a patent on some of his design work.

Andrew Brown received his $P h D$ in electrical engineering from the University of Michigan, Ann Arbor, in 1999. He is currently President of A. Brown Design, a small company focusing on contract design and consulting services in electrical

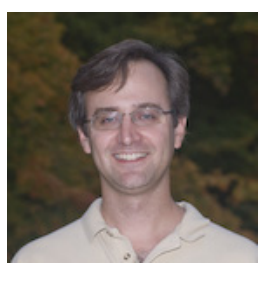
engineering using advanced or novel technologies. His current area of focus includes, but is not limited to, microwave and millimeter-wave tunable filters, MEMS based phase shifters, and resonant MEMS structures.

Louis Coryell is the Chief Technologist for SATCOM Antenna Research \& Development in the Space \& Terrestrial Communications Directorate of the US Army Communications \& Electronics Research, Development \& Engineering Center located at Fort Monmouth New Jersey. Mr. Coryell is

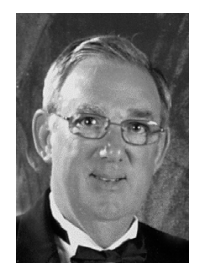
responsible for the technical aspects of all SATCOM Antenna systems and component development, including manufacturing technology efforts. He earned his Bachelor of Science in Electrical Engineering from Newark College of Engineering in 1968, a Master of Science in Electrical Engineering from Fairleigh Dickinson University in 1972 and a Master of Science in Business Administration from 
Fairleigh Dickinson University in 1985. Mr. Coryell has over 40 years of research and development experience in the areas of satellite communications systems, optical fiber communications systems, optical communications systems and related components. He has been the Program Manager for five Army manufacturing technology development efforts. 